\title{
Modelling Electronic Characteristic of InP/InGaAs Double Heterojunction Bipolar Transistor
}

\author{
Berrichi Yamina $^{1}$, Ghaffour Kherreddine ${ }^{2}$ \\ ${ }^{1}$ Faculty of Science, University of Tlemcen Algeria \\ ${ }^{2}$ Faculty of Technology, University of Tlemcen, Algeria
}

\section{Article Info \\ Article history: \\ Keyword: \\ Heterojunction \\ $\mathrm{InP} / \mathrm{InGaAs}$ \\ NPN \\ Transistor}

Received Feb 24, 2015

Revised Apr 10, 2015

Accepted Apr 27, 2015

\section{Corresponding Author:}

Berrichi Yamina

Faculty of Science, University of Tlemcen Algeria

Email: amina2010271@yahoo.fr

\begin{abstract}
In this paper, we are interested in studying $\mathrm{InP} / \mathrm{InGaAs}$ heterojunction bipolar transistor NPN type. First and for most we should describe the structure of our simulation, then, we ploted at room temperature: Energy band diagram, Gummel plot, $\mathrm{I}_{\mathrm{C}-} \mathrm{V}_{\mathrm{C}}$ characteristic and conduction bands for different values of $\mathrm{V}_{\mathrm{BE}}$. The simulation of this structure has demonstrated the validity of our model and the method of the simulation.
\end{abstract}

\section{INTRODUCTION}

The use of heterojunction to improve the performance of semiconductor devices is not a new concept; it was first suggested by William Shockley in 1951. At that time, however, semiconductor technology was not developed to the point where such novel concepts could be achieved in the laboratory [1].

At the name suggests, the semiconductor heterojunction is an idealized interface between two semiconductors. For device application such an interface has to be free of contaminants and the two semiconductors must generally be lattice matched so that no distortion of the epitaxial layers occurs to give rise unwanted defects within the layer. In these very special circumstances, the band diagrams of the separate materials can be joined continuously an engineered to produce some desired heterojunction behaviourThis new development has been called 'band gap engineering' and has provided a vehicle for a new understanding of semiconductor interface physics [1].

The performance advantage of HBTs is primarily derived from the use of wide bandgap emitters. If the emitter bandgap is larger than 'that in the base for an n-p-n HBT, the bandgap discontinuity sets up a barrier to the forward injection of electrons, resulting in a higher turn-on voltage for the emitter-base diode. More importantly, however, this discontinuity provides a barrier to the reverse injection of holes from the base into the emitter, increasing injection efficiency $\gamma$, significantly, as modeled by Equation (1) [2]:

$$
\gamma=\frac{\mathrm{N}_{\mathrm{E}}}{\mathrm{N}_{\mathrm{B}}} \exp \left(\frac{\Delta \mathrm{E}_{\mathrm{g}}}{\mathrm{kT}}\right)
$$

The introduction of an wide-gap emitter and collector to form a double heterojunction bipolar transistor (DHBT) offers several advantages over Homojunction Bipolar Transistors [3]:

- $\quad$ Higher $\mathrm{f}_{\mathrm{T}}$ and $\mathrm{f}_{\max }$ characteristic 
- $\quad$ increased breakdown voltage

- $\quad$ better performance under saturation operation Many parameters .

The objective of this paper is to establish the model and practical application of the method developed for describing electronic characteristics of $\mathrm{InP} / \mathrm{InGaAs}$ double heterojunction bipolar transistor and Future development of this work will extract the maximum and transition frequencies.

\section{CIRCIUT DIAGRAM OF HBT MODEL}

Figure 1 shows the schematic for the circuit diagram of HBT model.

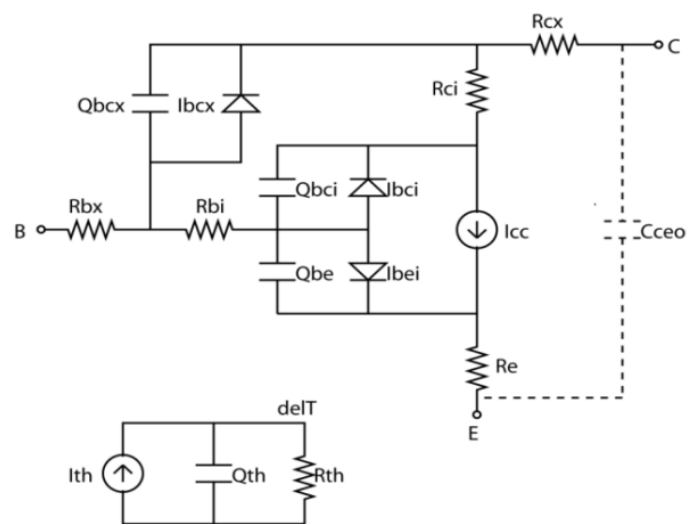

Figure 1. Circuit diagram for HBT [3]

The electron current injected from the emitter $\mathrm{I}_{\mathrm{nE}}$ to the base and the hole current $\mathrm{I}_{\mathrm{pE}}$ injected from the base to the emitter. For an HBT, only $\mathrm{I}_{\mathrm{nE}}$ contributes to the collector current (output current). A significant advantage of the HBT over the homojunction BJT is that the potential barrier for electrons is smaller than the potential barrier for holes, leading to an HBT injection efficiency that is close to unity [4].

Ideally, only the electrons injected into the base constitute the emitter current and that all these electrons are collected at the collector. We recover the classical relationship between the three currents transistor [5]:

$$
\mathrm{I}_{\mathrm{E}}=\mathrm{I}_{\mathrm{C}}+\mathrm{I}_{\mathrm{B}}
$$

\section{RESEARCH METHOD}

Years of research into device physics have resulted in a mathematical model that operates on any semiconductor device [6]. The model consists of a set of fundamental equations which link together the electrostatic potential and the carrier densities, within some simulation domain. These equations, which are solved inside any general purpose device simulator, have been derived from Maxwell's laws and consist of Poisson's Equation the continuity equations and the transport equations Poisson's Equation relates variations in electrostatic potential to local charge densities. The continuity and the transport equations describe the way that the electron and hole densities evolve as a result of transport processes, generation processes, and recombination processes.

Different combinations of models will require solving up the equations of the transport, the simplest model is « the drift diffusion transport model » [7].

Drift-diffusion theory to calculate carrier transport over a heterojunction, in these model current expressions for electrons and holes denseties is given by:

$$
\begin{aligned}
& \mathrm{J}_{\mathrm{n}}=\mathrm{qn}(x) \mu_{\mathrm{n}} \mathrm{E}(x)+\mathrm{qD}_{\mathrm{n}} \frac{\mathrm{dn}}{\mathrm{d} x} \\
& \mathrm{~J}_{\mathrm{p}}=\mathrm{qp}(x) \mu_{\mathrm{p}} \mathrm{E}(x)-\mathrm{qD}_{\mathrm{p}} \frac{\mathrm{dp}}{\mathrm{d} x}
\end{aligned}
$$


Where $\mathrm{q}$ is used to indicate the absolute value of the electronic charge, $\mu(\mathrm{E})$ is mobility and $\mathrm{D}$ is diffusion coefficient.

The (3), (4) equations to be solved are Poisson's equation and the current continuity equations for electrons and holes.

Continuity equations are:

$$
\begin{aligned}
& \frac{\partial \mathrm{n}}{\partial t}=\frac{1}{\mathrm{q}} \nabla \cdot \mathrm{J}_{\mathrm{n}}+\mathrm{U}_{\mathrm{n}} \\
& \frac{\partial \mathrm{p}}{\partial t}=\frac{1}{\mathrm{q}} \nabla \cdot \mathrm{J}_{\mathrm{p}}+\mathrm{U}_{\mathrm{p}}
\end{aligned}
$$

Poisson's equation is:

$$
\nabla \cdot \varepsilon \nabla V=-\left(p-n+N_{D}^{+}-N_{A}^{-}\right)
$$

Equation (3) through (7) constitute a system of five equations in five unknowns (n, p, $J_{n}, J_{p}$ and V) and given appropriate boundary conditions, can be used to analyze the carrier concentrations, currents and fields in an arbitrary device structure.However, we also need to specify the other parameters (mobility, net recombination rate, ect) and how they depend on the material properties, carrier densities and local potential and field.

The resolution of the above equations have based on the mesh, treatment of mesh points for the continuity equations at a heterojunctions is solved by the Newton method.

\section{RESULTS AND ANALYSIS}

\subsection{Structure of Simulation}

The layer structure of the InP/InGaAs DHBT is shown in Figure 2.

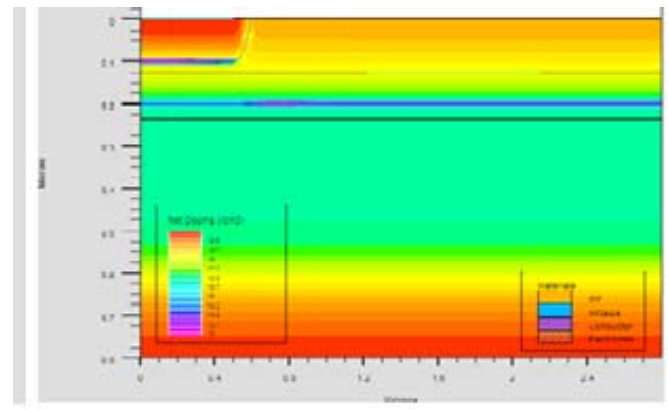

Figure 2. Structure of InP / InGaAs double heterojunction bipolar transistor

As shown on table I, the transistor consists of $0,125 \mu \mathrm{m}$ thick $6 \times 10^{19} \mathrm{~cm}^{-3} \mathrm{n}$-InP emitter, $0,09 \mu \mathrm{m}$ thick $3 \times 10^{18} \mathrm{~cm}^{-3}$ p-InGaAs base, $0,1 \mu \mathrm{m}$ thick $4 \times 10^{17} \mathrm{~cm}^{-3} \mathrm{n}$ - InP sub-collector and $0,4944 \mu \mathrm{m}$ thick $8 \times 10^{18}$ $\mathrm{cm}^{-3} \mathrm{n}$ - InP collector.

Table 1. The parametre deffinition of mesh and doping

\begin{tabular}{lcc}
\hline \multicolumn{1}{c}{ Electrodes } & Dopage $\left(\mathrm{cm}^{-3}\right)$ & Thickness $(\mu \mathrm{m})$ \\
\hline Emitter $(\mathrm{InP})$ & $6 \times 10^{19}$ & 0.125 \\
$\begin{array}{l}\text { Base } \\
\text { (InGaAs) }\end{array}$ & $3 \times 10^{18}$ & 0.090 \\
$\begin{array}{l}\text { Sub-Collector } \\
\text { (InP) }\end{array}$ & $4 \times 10^{17}$ & 0.100 \\
Cllector $(\mathrm{InP})$ & $8 \times 10^{18}$ & 0.4944 \\
\hline
\end{tabular}




\subsection{Heterojunction}

Figure 3 shows the evolution of energy valence and conduction bands.

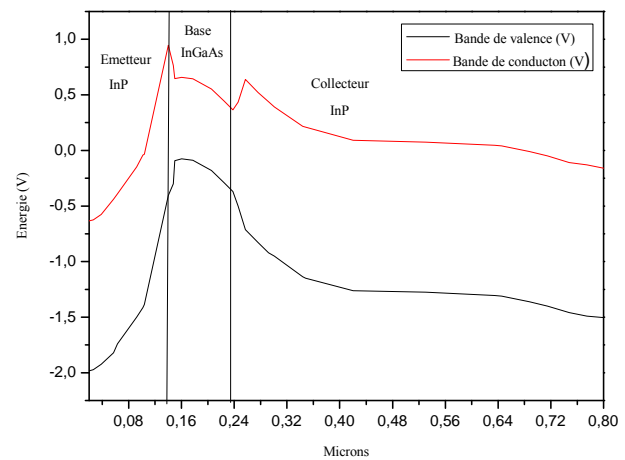

Figure 3. Energy band diagram of InP / InGaAs double heterojunction bipolar transistor

Once the two materials are jointed to from heterojuntion, the energy band diagram changes. The InGaAs has a bandgap of only $(0,75 \mathrm{eV}[8])$, is used for the base and the wider bandgap material of InP $(1,35 \mathrm{eV}[8])$ is used for the emitter and collector material. The thermal equilibrium band diagram of the InPInGaAs -InP is shows in the Figure 3.

Obviously, the potential spike at E-B junction can be completely eliminated due to the employments of a heavy doped as well as thin n-InP emitter layer, even at $\mathrm{V}_{\mathrm{EB}} \neq 0 \mathrm{~V}$. The thin $\mathrm{n}$-InP emitter layer may help to promote the energy band at emitter side and increase the effective potential barrier for holes. Thus, the transistor actions with high emitter injection efficiency and current gain are expectable.

\subsection{Conduction Bands Diagram for Different Value of $V_{B E}$.}

Figure 4 shows conduction bands diagram for different value of $\mathrm{V}_{\mathrm{BE}}$.

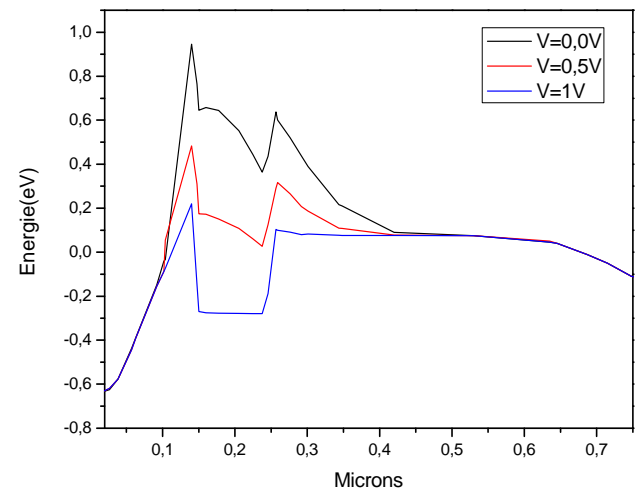

Figure 4. Conduction bands diagram of InP/InGaAs double heterojunction bipolar transistor for different value of $\mathrm{V}_{\mathrm{BE}}$

Simulation of conduction band diagram for a InP/InGaAs doubles heterojunction bipolar transistor with different values of $\mathrm{V}_{\mathrm{BE}}$. The figure 4 shows the decrease conduction band barrier at the emitter-base junction, when we increase the value of the voltage $\mathrm{V}_{\mathrm{EB}}$.

\subsection{Gummel Plot}

The Figure 5 shows the collector and base current of InP / InGaAs double heterojunction bipolar transistor, biased in the forward active mode of operation with $\mathrm{V}_{\mathrm{C}}=1 \mathrm{~V}$, as a function of the base-emitter voltage. This type of plot is also called a Gummel plot. 


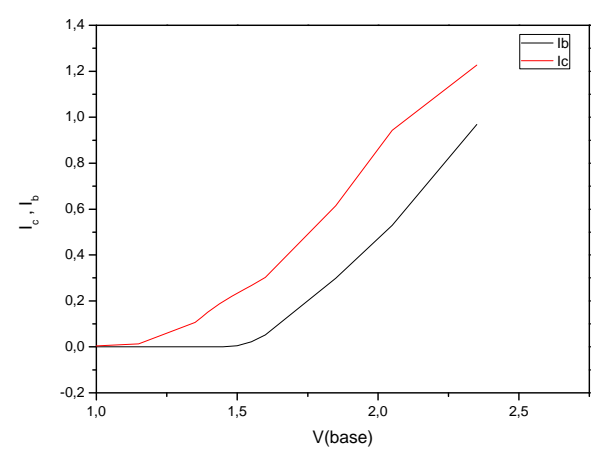

Figure 5. Gummel plot of InP / InGaAs double heterojunction bipolar transistor

This plot is very useful in device characterization because it reflects on the quality of the emitterbase junction while the base-collector bias, $\mathrm{V}_{\mathrm{BC}}$, is kept at a constant.

A number of other device parameters can be garnered either quantitatively or qualitatively directly from the Gummel plot [9]:

- $\quad$ The common-emitter current gain $\beta$ and the common-base current gain $\alpha$.

- $\quad$ Base and collector ideality factors $\eta$.

- Series resistances and leakage currents.

Gummel plot can be divided into three distinct regions:

$0 \mathrm{~V}<\mathrm{V}_{\mathrm{BE}}<3 \mathrm{~V}$ : This region corresponds to the normal bias conditions and ideal, the base and collector current is dominated by the diffusion current (current of electrons injected from the emitter into the base).

$\mathrm{V}_{\mathrm{BE}}<1,2 \mathrm{~V}$ : Recombination currents are added to the diffusion current.

$\mathrm{V}_{\mathrm{BE}}>3$ : The base and collector currents are slightly reduced because of the phenomena at high doses (Kirk effect).

\subsection{Ic-Vce Characteristics}

Figure 6 shows a typical output of InP/InGaAs double heterojunction bipolar transistor characteristic, which is the collector current Ic versus the collector-emitter voltage at constant base current.

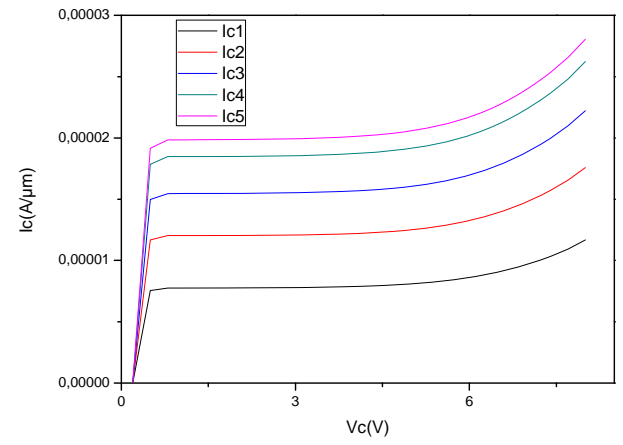

Figure 6. Ic-Vce characteristics of InP/InGaAs double heterojunction bipolar transistor

I-V plot can be divided into three distinct regions:

- Region $1\left(0 \mathrm{~V}<\mathrm{V}_{\mathrm{BE}}<0,2 \mathrm{~V}\right)$ : is the non- linear region due to non-exponential behaviour of diodes at low voltages (leakage currents amongst other factors).

- $\quad$ Region $2\left(0,2 \mathrm{~V}<\mathrm{V}_{\mathrm{BE}}<0,9 \mathrm{~V}\right)$ : is the linear region

- Region $3\left(0,9 \mathrm{~V}<\mathrm{V}_{\mathrm{BE}}<10 \mathrm{~V}\right)$ : the current is limited by the series resistance. 


\section{CONCLUSION}

In this paper, we studied InP/InGaAs doubles heterojunction bipolar transistor device. Modeling and simulation were performed by using ATLAS-TCAD simulator. Our simulations prove the quality and validity of our model for modelling electronic characteristic of the $\mathrm{InP} / \mathrm{InGaAs}$ heterojunction bipolar transistor.

\section{ACKNOWLEDGEMENTS}

We thank the members the Unity of Research "Materials and Renewable Energies", Faculty of Science, University of Abou-bekr Belkaid, Tlemcen, Algeria.

\section{REFERENCES}

[1] D.V. Morgan, Robin H. Villiams, Physics and Technology of Heterojunction Devices published by: Peter peregrines Ltd, London, United Kingdom, 1991.

[2] S.M. Sze, Physics of Semiconductor Devices (Second Edition), Wiley-Interscience, 1981, pp. 142.

[3] Tom K. Johansen, Virginie Nodjiadjim, Jean-Yves Dupuy, Agnieszka konczykowska, Small- and Large- Signal Modeling for Submicron InP/InGaAs DHBT's, DTU Electrical Engineering, Electromagnetic Systems Group, Technical University of Denmark, 2012.

[4] Kwok-Leung Chan, High resolution thermal imaging for electrical and optical characterization of electronic and photonic devices, University of Michigan, 2007.

[5] Florence Brossard, Epitaxies $\mathrm{Si} / \mathrm{SiGe}(\mathrm{C})$ pour transistors bipolaires avancés, Université Joseph-Fourier- Grenoble. French, 2007.

[6] M.R. Pinto, Conor S. Rafferty, and Robert W. Dutton, PISCES2 - Poisson and Continuity Equation Solver, Stanford Electronics Laboratory Technical Report, Stanford University, September 1984.

[7] S. Selberherr, Analysis and Simulation of Semiconductor Devices, Springer-Verlag, Wien-New York, 1984.

[8] Subhra Chowdhury, Sukla Basu, Effect of device parameters on current voltage characteristics and current gain of InP/InGaAs HBTs, Electronics and Communication Engineering Department, Kalyani Government Engineering College Kalyani , India journal of Electron Devices, Vol. 9, 2011, pp. 362-366.

[9] A.S. Zoolfakar, N.A. Shahrol, Modelling of NPN Bipolar Junction Transistor Characteristics Using Gummel Plot Technique, International Conference on Intelligent Systems, Modelling and Simulation (ISMS), 2010, p. 396-400. 